\title{
Two spotted and magnetic early B-type stars in the young open cluster NGC 2264 discovered by MOST and ESPaDOnS ${ }^{\star}, \star \star$
}

\author{
L. Fossati ${ }^{1}$, K. Zwintz ${ }^{2}$, N. Castro ${ }^{1}$, N. Langer ${ }^{1}$, D. Lorenz ${ }^{3}$, F. R. N. Schneider ${ }^{1}$, R. Kuschnig ${ }^{3}$, J. M. Matthews ${ }^{4}$, \\ E. Alecian ${ }^{5,6}$, G. A. Wade ${ }^{7}$, T. G. Barnes ${ }^{8}$, and A. A. Thoul ${ }^{9}$
}

\author{
1 Argelander-Institut für Astronomie der Universität Bonn, Auf dem Hügel 71, 53121 Bonn, Germany \\ e-mail: Ifossati@astro.uni-bonn.de \\ 2 Instituut voor Sterrenkunde, K. U. Leuven, Celestijnenlaan 200D, 3001 Leuven, Belgium \\ 3 University of Vienna, Department of Astronomy, Türkenschanzstrasse 17, 1180 Vienna, Austria \\ ${ }^{4}$ Department of Physics and Astronomy, University of British Columbia, 6224 Agricultural Road, Vancouver V6T 1Z1, Canada \\ 5 UJF-Grenoble 1/CNRS-INSU, Institut de Planétologie et d'Astrophysique de Grenoble (IPAG) UMR 5274, 38041 Grenoble, France \\ ${ }^{6}$ LESIA-Observatoire de Paris, CNRS, UPMC Univ., Univ. Paris-Diderot, 5 place Jules Janssen, 92195 Meudon Principal Cedex, \\ France \\ 7 Department of Physics, Royal Military College of Canada, PO Box 17000, Stn Forces, Kingston K7K 7B4, Canada \\ 8 The University of Texas at Austin, McDonald Observatory, 82 Mt. Locke Rd., McDonald Observatory, Texas, 79734, USA \\ 9 Institut d'Astrophysique et de Géophysique, Université de Liège, 17 Allée du 6 Août, 4000 Liège, Belgium
}

Received 7 December 2013 / Accepted 15 January 2014

\begin{abstract}
Star clusters are known as superb tools for understanding stellar evolution. In a quest for understanding the physical origin of magnetism and chemical peculiarity in about $7 \%$ of the massive main-sequence stars, we analysed two of the ten brightest members of the $\sim 10 \mathrm{Myr}$ old Galactic open cluster NGC 2264, the early B-dwarfs HD 47887 and HD 47777 . We find accurate rotation periods of 1.95 and 2.64 days, respectively, from MOST photometry. We obtained ESPaDOnS spectropolarimetric observations, through which we determined stellar parameters, detailed chemical surface abundances, projected rotational velocities, and the inclination angles of the rotation axis. Because we found only small $\left(<5 \mathrm{~km} \mathrm{~s}^{-1}\right)$ radial velocity variations, most likely caused by spots, we can rule out that HD 47887 and HD 47777 are close binaries. Finally, using the least-squares deconvolution technique, we found that both stars possess a large-scale magnetic field with an average longitudinal field strength of about $400 \mathrm{G}$. From a simultaneous fit of the stellar parameters we determine the evolutionary masses of HD 47887 and HD 47777 to be $9.4_{-0.7}^{+0.6} M_{\odot}$ and $7.6_{-0.5}^{+0.5} M_{\odot}$. Interestingly, HD 47777 shows a remarkable helium underabundance, typical of helium-weak chemically peculiar stars, while the abundances of HD 47887 are normal, which might imply that diffusion is operating in the lower mass star but not in the slightly more massive one. Furthermore, we argue that the rather slow rotation, as well as the lack of nitrogen enrichment in both stars, can be consistent with both the fossil and the binary hypothesis for the origin of the magnetic field. However, the presence of two magnetic and apparently single stars near the top of the cluster mass-function may speak in favour of the latter.
\end{abstract}

Key words. stars: fundamental parameters - stars: early-type - stars: individual: HD 47887 - stars: individual: HD 47777 open clusters and associations: individual: NGC 2264 - stars: magnetic field

\section{Introduction}

Understanding the evolution of stars is a prerequisite for understanding galaxies and galaxy evolution. In intermediate-mass and massive stars, stellar rotation and binarity (Langer 2012; Maeder \& Meynet 2012; Sana et al. 2012) were identified as playing important roles in stellar evolution, which is currently studied in considerable detail. The role of strong magnetic fields is still poorly understood (Langer 2012), even though they have

* Based on data from the MOST satellite, a Canadian Space Agency mission, jointly operated by Microsatellite Systems Canada Inc. (MSCI), formerly part of Dynacon, Inc., the University of Toronto Institute for Aerospace Studies and the University of British Columbia with the assistance of the University of Vienna.

$\star \star$ Based on observations obtained at the Canada-France-Hawaii Telescope (CFHT) which is operated by the National Research Council of Canada, the Institut National des Science de l'Univers of the Centre National de la Recherche Scientifique of France, and the University of Hawaii. long been known to be present in some intermediate-mass and massive stars.

It has recently been established that the incidence of largescale magnetic fields in massive and intermediate-mass stars is of the order of 7\% (Wade et al. 2013). From the intermediatemass stars we know that their magnetic fields are either strong $(>100 \mathrm{G})$ or very weak $(<1 \mathrm{G})$, that is, they show a true bimodality (Auriere et al. 2007). While for intermediate-mass stars, magnetic fields give rise to strong chemical peculiarities (Ap, Bp stars), a clear-cut correlation of the presence of a strong magnetic field and abnormal chemical surface abundances in massive stars has not been yet established (Martins et al. 2012).

One of the most pressing questions at present is to identify the origin of the strong magnetic fields in intermediate-mass and massive stars. It has been suggested that the magnetic fields might be "fossil", that is, inherited from the birth molecular cloud that formed the star, or produced by a pre-main-sequence dynamo (e.g., Moss 2001). Alternatively, the magnetic fields might be produced in the course of a strong binary interaction, 


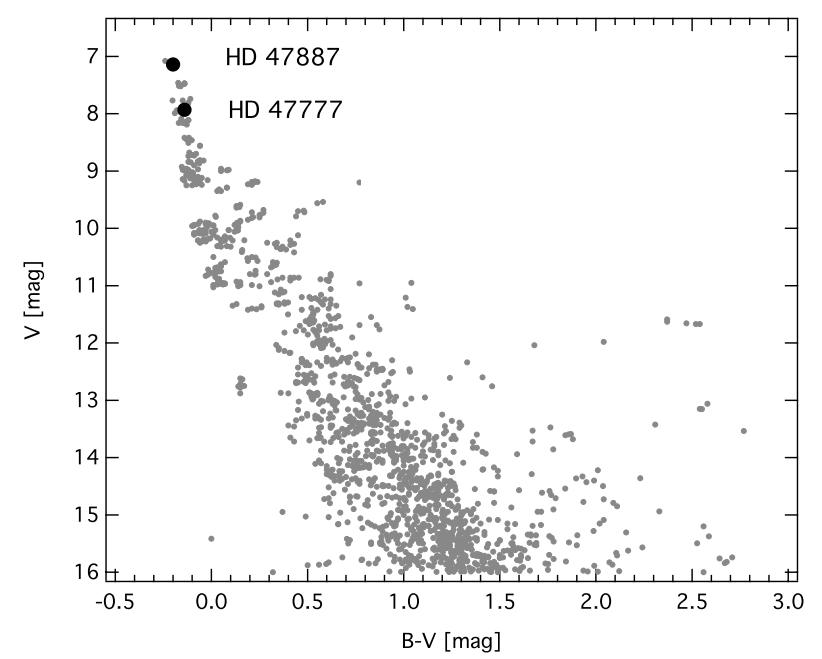

Fig. 1. Colour-magnitude diagram (CMD) of the open cluster NGC 2264 in the Johnson $B$ and $V$ filters (grey points, source: WEBDA database of open clusters - Mermilliod \& Paunzen 2003) showing the positions of HD 47887 and HD 47777 (Walker 1956).

for instance, through a stellar binary merger (e.g., Ferrario et al. 2009; Grunhut et al. 2008). Stars that produce magnetic fields in a pre-main-sequence merger will be difficult to distinguish from those that inherited their field from an earlier stage. However, main-sequence binary interaction will lead to a rejuvenation of the mass gainer (or merger product). Therefore, it may be useful to investigate magnetic stars in star clusters to test these ideas.

NGC $2264\left(\alpha_{2000}=6^{\mathrm{h}} 41^{\mathrm{m}}, \delta_{2000}=+9^{\circ} 53^{\prime}\right)$ was studied frequently in the past using various instruments in different wavelength ranges (from X-ray to radio wavelengths) from space and from the ground (e.g., Sung et al. 1997; Flaccomio et al. 2006; Sung et al. 2009; Zwintz et al. 2009). The cluster is located in the Monoceros OB1 association about $30 \mathrm{pc}$ above the Galactic plane and has a diameter of $\sim 39$ arcmin. The age of NGC 2264 can only be determined with a rather large uncertainty as its main sequence consists only of a few massive B-type stars (no mainsequence O-type star is present in the cluster field of view), while stars of later spectral types are still in their pre-main-sequence phase (e.g., Zwintz et al. 2013a,b). Different cluster ages have therefore been reported in the literature and range from 3 to $14 \mathrm{Myr}(\log t=6.5-7.15)$ (e.g., Dambis 1999; Spassova \& Beav 1985). Figure 1 shows the colour-magnitude (CMD) diagram of the NGC 2264 open cluster in Johnson $B$ and $V$ photometry. The stars HD 47887 (NGC 2264 178) and HD 47777 (NGC 2264 83), two of the brightest, hottest, and most massive stars in the cluster, indicated in Fig. 1 as black circles, are the subject of this work. Their position in the sky is shown in Fig. 2.

With a $V$ magnitude of 7.14 (Walker 1956), HD 47887 is the second-brightest object in the NGC 2264 open cluster and was classified as B1.5V by Morgan et al. (1965). Flaccomio et al. (2000) detected HD 47887 in the X-rays with the ROSAT HRI instrument, obtaining a maximum X-ray luminosity of $\log L_{\mathrm{X}}\left[\mathrm{erg} \mathrm{s}^{-1}\right]<30.26\left(\log L_{\mathrm{X}} / L_{\text {bol }}<-7.1\right)$.

HD 47777 ( $V=7.93$ mag Walker 1956) is a known magnetic early B-type star (B2IV Morgan et al. 1965), listed in the Renson \& Manfroid (2009) catalogue of chemically peculiar stars. For this star, Bychkov et al. (2009) reported an average quadratic field modulus of $355 \mathrm{G}$ on the basis of the analysis of metallic lines, while, making use of the least-squares deconvolution technique (LSD; Donati et al. 1997), Alecian et al. (2009) presented a firm magnetic field detection. Flaccomio et al. (2000) detected

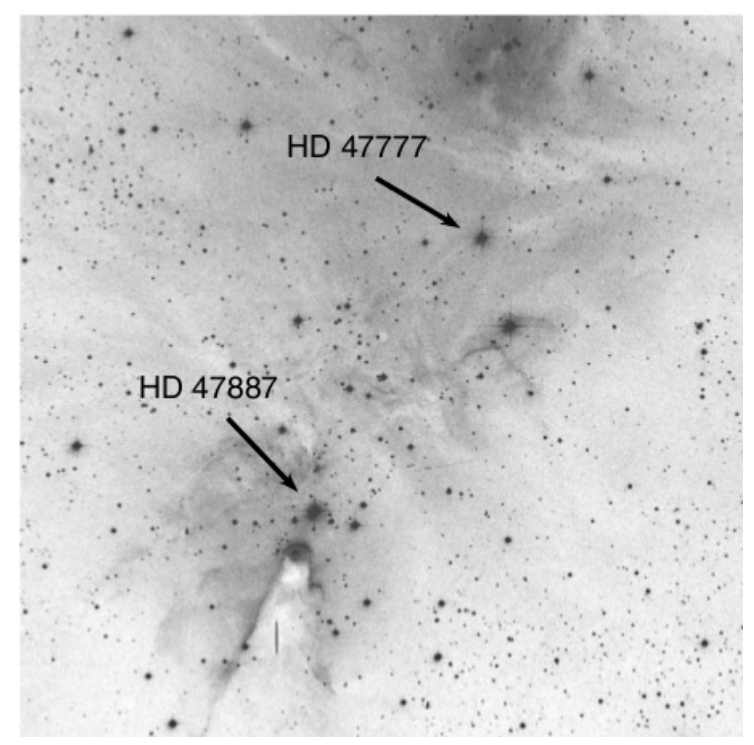

Fig. 2. DSS image of a $30 \times 30 \mathrm{arcmin}^{2}$ region showing the positions of HD 47887 and HD 47777 in the sky. The "elephant trunk" visible at the bottom of the image is probably caused by the intense radiation of the massive cluster-stars falling onto a dense region of the interstellar medium. The geometry of the elephant trunk suggests that HD 47887 plays an important role in its formation.

HD 47777 in X-rays with the ROSAT HRI instrument, obtaining an X-ray luminosity of $\log L_{\mathrm{X}}\left[\mathrm{erg} \mathrm{s}^{-1}\right]=30.69\left(\log L_{\mathrm{X}} / L_{\mathrm{bol}} \simeq\right.$ $-6.3)$, in agreement with the later measurement of Dahm et al. (2007) of $\log L_{X}\left[\mathrm{erg} \mathrm{s}^{-1}\right]=30.65 \pm 0.04$.

Zwintz et al. (2009) presented light curves of both HD 47887 and HD 47777 obtained with the MOST satellite (Walker et al. 2003 ) in 2006, showing a clear rotationally modulated variability. The observed rotational modulation, which is caused by spots, led Zwintz et al. (2009) to classify HD 47887 and HD 47777 as Bp stars despite of the lack of spectroscopic data.

Following these findings, we obtained additional photometric and spectropolarimetric data to perform a detailed analysis of the two stars.

\section{Photometric analysis}

\subsection{MOST observations and data reduction}

The Canadian micro-satellite MOST (Walker et al. 2003) carries a $15 \mathrm{~cm}$ Rumak Maksutov telescope that feeds a CCD photometer through a single custom broadband filter in the 3500-7500 wavelength range. The MOST space telescope was launched on June 30, 2003, hence is in its eleventh year of highly successful operation. Three types of photometric data can be supplied simultaneously for multiple targets in a given field: Fabry imaging, direct imaging, and Guide Star Photometry data.

HD 47887 and HD 47777 are included in the MOST observing runs on the young open cluster NGC 2264. Because of the magnitude range $(7<V<12 \mathrm{mag}$ ) and the large number of targets, the cluster was observed in the open field of the MOST Science CCD in Guide Star Photometry mode. MOST observed the cluster NGC 2264 twice: the first time in 2006 and the second time in 2011/2012 as part of the CSI2264 (coordinated synoptic investigation of NGC 2264) project together with the space telescopes CoRoT (Baglin 2006), Spitzer (Werner et al. 2004) and Chandra (Weisskopf et al. 2002). During both MOST observing runs two fields of observations were chosen and observed in 

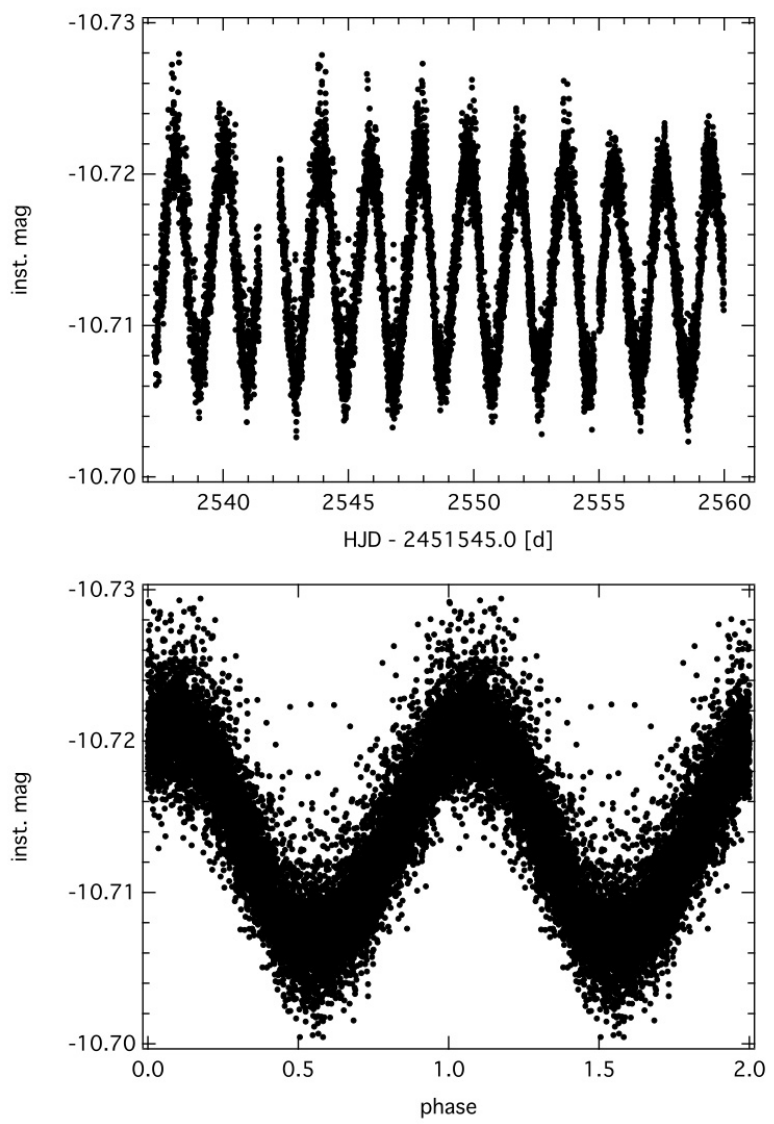

Fig. 3. Top panel: MOST 2006 light curve of HD 47887. Bottom panel: light curve phased with a period of 1.949 days.

alternating halves of each 101 min orbit to increase the number of targets. In 2006 the fields were named field A and field B, in 2011/12 the fields were named field 1 and field 2 .

MOST observed NGC 2264 from December 7, 2006, to January 3, 2007. On-board exposures were $1.5 \mathrm{~s}$ long to satisfy the cadence of the Guide Star ACS operations. Sequences of 16 consecutive exposures were stacked on board, sampled about twice per minute. HD 47887 was included in field A with a total time base of 22.72 days, while HD 47777 was observed in field B with a total time base of 22.77 days. A detailed description of the 2006 MOST data of NGC 2264 can be found in Zwintz et al. (2009).

During the second MOST run on NGC 2264 in 2011/2012 the on-board exposures were $3.01 \mathrm{~s}$ long, and the integration time was $51.17 \mathrm{~s}$ as 17 consecutive images were stacked on board. HD 47777 was observed in field 1 with a time base of 38.77 days. Because of a slightly different pointing of MOST in 2011/12 compared with 2006, no second light curve of HD47887 is available.

For the reduction of the MOST Guide Star photometry of all stars in the cluster, we used the method developed by Hareter et al. (2008; for more details see Zwintz et al. 2009). Consequently, the 2006 light curve of HD 47887 has 10531 data points (see Fig. 3) with a resulting Nyquist frequency of $1362.23 \mathrm{~d}^{-1}$; the 2006 data set of HD 47777 consists of 17698 data points (top-left panel in Fig. 4), and the 2011/12 light curve has 16688 data points (top-right panel in Fig. 4), corresponding to Nyquist frequencies of $1416.73 \mathrm{~d}^{-1}$ and $721.34 \mathrm{~d}^{-1}$, respectively. Note that the $2011 / 12$ light curve has fewer data points than the 2006 light curve although the time
Table 1. Characteristics of the MOST observations of HD 47777 and HD 47887.

\begin{tabular}{llrrcrc}
\hline \hline Data set & Star & $\begin{array}{r}\text { Points } \\
\#\end{array}$ & $\begin{array}{r}\text { Tbase } \\
{[\mathrm{d}]}\end{array}$ & $\begin{array}{r}1 / T \\
{\left[\mathrm{~d}^{-1}\right]}\end{array}$ & $\begin{array}{r}f_{\text {Nyquist }} \\
{\left[\mathrm{d}^{-1}\right]}\end{array}$ & $\begin{array}{r}\text { noise } \\
{[\mathrm{ppm}]}\end{array}$ \\
\hline 2006 & HD 47777 & 17698 & 22.77 & 0.044 & 1416.73 & 48.5 \\
& HD 47887 & 10531 & 22.72 & 0.044 & 1362.23 & 45.9 \\
$2011 / 12$ & HD 47777 & 16688 & 38.77 & 0.026 & 721.34 & 33.9 \\
\hline
\end{tabular}

Notes. This table lists: data set, number of data points used for the analysis, time base, Rayleigh frequency resolution (1/T), Nyquist frequency, and noise level of the residuals (noise ${ }_{\mathrm{res}}$ ).

base is significantly longer. The reason is that in 2011/12 HD 47777 was observed in the part of the orbit affected by worse stray-light conditions than in 2006, hence more data points had to be discarded in the reduction.

Table 1 presents an overview of the properties of the MOST observations. Figures 3 and 4 show the MOST light curves of the two stars.

\subsection{Frequency analysis}

For the frequency analyses, we used the software package Period04 (Lenz \& Breger 2005), which combines Fourier and least-squares algorithms. Frequencies were then prewhitened and considered to be significant if their amplitudes exceeded four times the local noise level in the amplitude spectrum (i.e., 4 S/N; Breger 1993; Kuschnig et al. 1997).

We additionally verified the analysis using the SigSpec software (Reegen 2007). SigSpec computes significance levels for amplitude spectra of time series with arbitrary time sampling. The probability density function of a given amplitude level is solved analytically and the solution includes dependences on the frequency and phase of the signal.

We found a single intrinsic frequency for the two stars which in both cases was interpreted to be due to rotational modulation of the star. For HD 47777 we have observations from two epochs that were first analysed separately and the results compared with each other. In both data sets a frequency, $F$, of $0.3787 \mathrm{~d}^{-1}$ (i.e., $4.383 \mu \mathrm{Hz}$ ) was identified (see Table 2 and Fig. 5), corresponding to a rotation period of 2.641 days. Twice $F$ and three times $F$ can also be identified in each of the two data sets separately. The difference in $F$ obtained from the 2006 data and from the 2011/12 data is less than the individual errors on the frequency, hence the frequency does not significantly change within a time span of about five years. The differences in amplitude are also relatively small and amount to $0.111 \mathrm{mmag}$. In a second step the two data sets were combined to determine a common solution, obtaining the same frequency $F$ as for the separated analysis. Because frequency and amplitude of the variability do not change over more than five years, the structures causing the rotational modulation of the light curve must be very stable during this period of time. Table 2 lists the frequency and amplitude values for HD 47777 obtained separately for the two years to illustrate this stability.

For HD 47887 we found the frequency $F$ at $0.513 \mathrm{~d}^{-1}$ (i.e., $5.94 \mu \mathrm{Hz})$, as can be seen in the amplitude spectrum shown in the upper panel of Fig. 6 . Its multiples, that is, $1 / 2 F, 2 F$, and $3 F$, are also present at lower amplitudes (see bottom panel of Fig. 6) and are strong indicators for rotational modulation caused by a non-uniform stellar surface. 

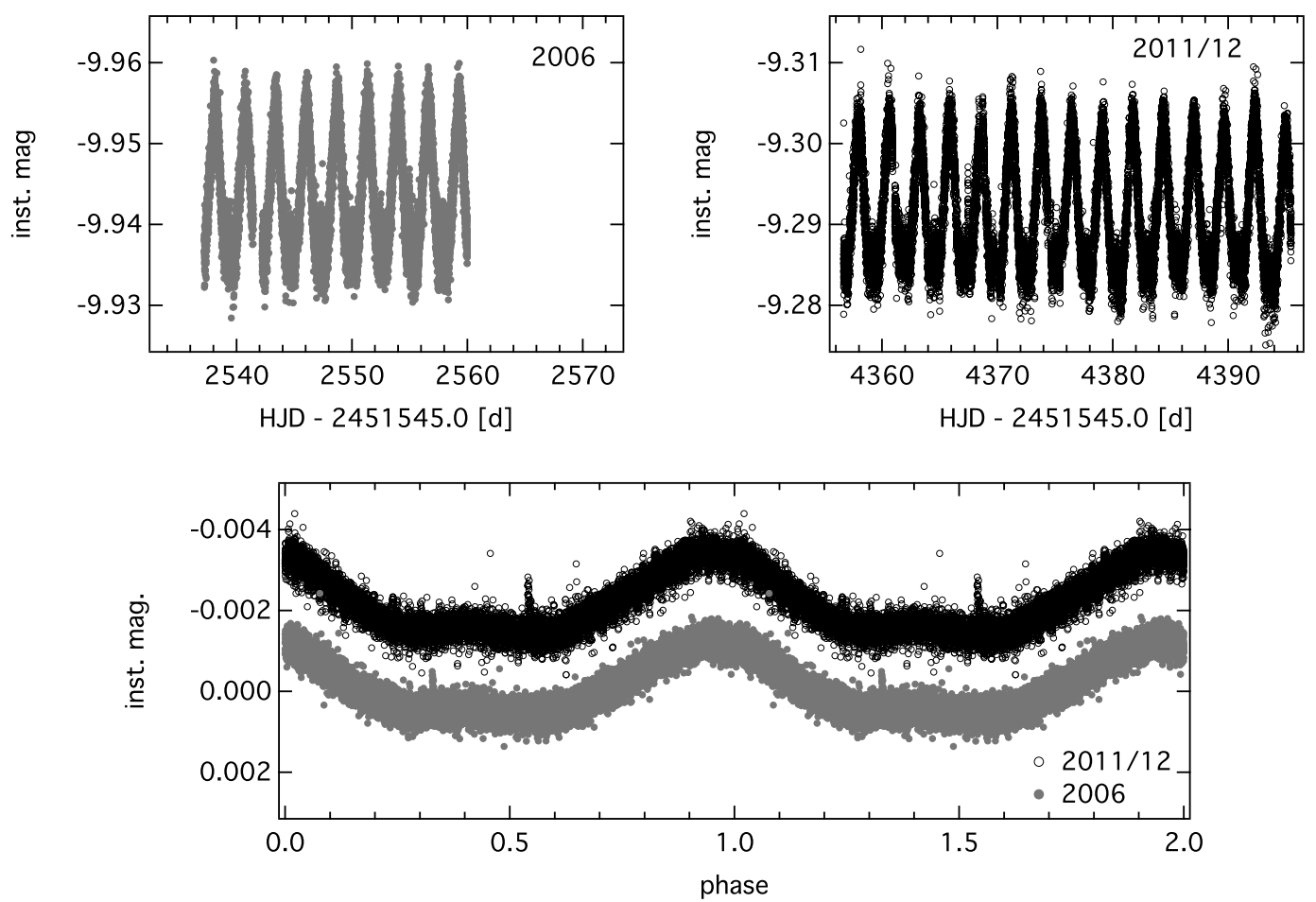

Fig. 4. MOST light curve and phase plot for HD 47777: the 2006 data set (grey dots, top-left panel), the 2011/12 data set (black circles, top-right panel), phase plot with a period of 2.641 days (bottom panel). Note that the $2011 / 12$ data set was shifted for clarity.

Table 2. Results of the frequency analysis of HD 47777 and HD 47887.

\begin{tabular}{lllrrrrr}
\hline \hline Star & Data set & \multicolumn{2}{c}{ Freq. } & $\begin{array}{r}\text { Amp } \\
{\left[\mathrm{d}^{-1}\right]}\end{array}$ & $\begin{array}{r}\text { Period } \\
{[\mu \mathrm{Hz}]}\end{array}$ & $S / N$ & Sig \\
{$[\mathrm{mmag}]$} & {$[$ days $]$} & & \\
\hline HD 47777 & 2006 & $0.3786(8)$ & $4.382(9)$ & 8.741 & 2.640 & 8.87 & 3246.02 \\
& $2011 / 12$ & $0.3787(5)$ & $4.383(5)$ & 8.852 & 2.641 & 12.73 & 3027.30 \\
HD 47887 & 2006 & $0.513(1)$ & $5.94(1)$ & 7.060 & 1.949 & 12.19 & 1857.05 \\
\hline
\end{tabular}

Notes. This table lists: frequencies, amplitudes, phases, signal-to-noise values $(\mathrm{S} / \mathrm{N})$ and SigSpec significances (sig). The respective last-digit errors of the frequencies computed according to Kallinger et al. (2008) are given in parentheses. The second line for HD 47777 shows the parameters for $F$ determined from the 2011/12 data set to illustrate its stability.

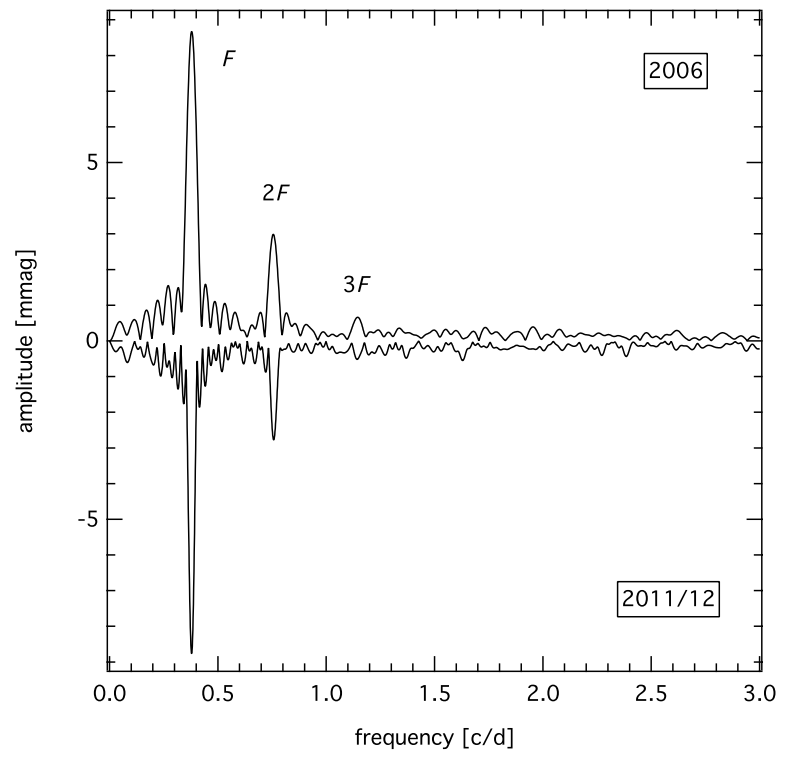

Fig. 5. Amplitude spectrum of $\mathrm{HD} 47777$ where $F=0.3787 \mathrm{~d}^{-1}$ is marked; twice $F$ and three times $F$ are also marked: 2006 data are pointing upwards, 2011/12 data downwards.

\section{Spectroscopic analysis and stellar parameters}

\subsection{Observations and cluster membership}

Spectra of HD 47887 and of HD 47777 were obtained on 2012 January 31 (HJD: 2455957.96662) and February 3rd (HJD: 2455 960.74013), respectively, with the ESPaDOnS spectropolarimeter of the Canada-France-Hawaii Telescope (CFHT). ESPaDOnS consists of a table-top, cross-dispersed échelle spectrograph fed by a double optical fiber directly from a Cassegrainmounted polarisation analysis module. Both Stokes $I$ and $V$ spectra were obtained throughout the 3700-10400 $\AA$ spectral range at a resolving power of about 65000 . The spectra were reduced using the Libre-ESpRIT reduction pipeline (Donati et al. 1997). The signal-to-noise ratio $(\mathrm{S} / \mathrm{N})$ per $1.8 \mathrm{~km} \mathrm{~s}^{-1}$ spectral pixel at $\lambda \sim 5000 \AA$ is 327 and 409, respectively for HD 47887 and HD 47777.

The spectra of the two stars were normalised by fitting a low-order polynomial to carefully selected continuum points. For each star, we determined the radial velocity $\left(v_{r}\right)$ by fitting a Gaussian to the Stokes I LSD profile (see Sect. 3.3). From the ESPaDOnS spectra we obtained $v_{r}=25.2 \pm 0.3 \mathrm{~km} \mathrm{~s}^{-1}$ and $v_{r}=26.7 \pm 0.9 \mathrm{~km} \mathrm{~s}^{-1}$ for HD 47887 and HD 47777, respectively. For HD 47887, Abt (1970) obtained a single radial 

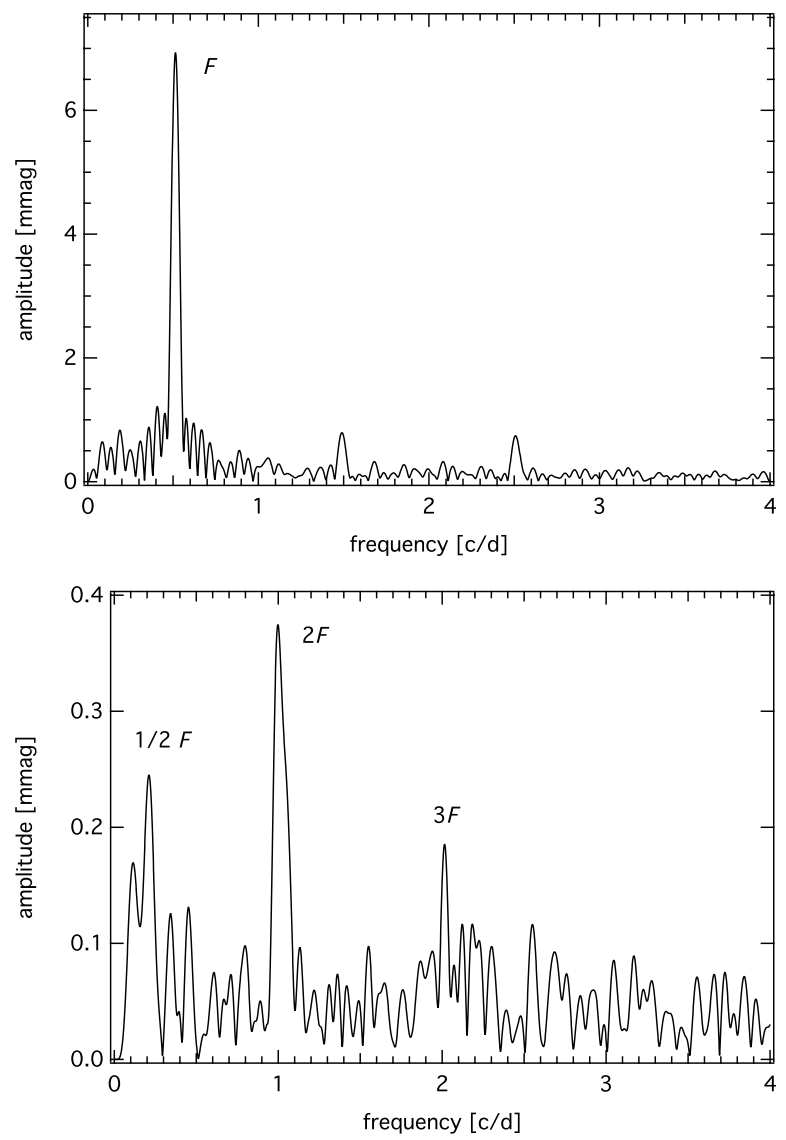

Fig. 6. Top panel: original amplitude spectrum of HD 47887 where $F=$ $0.513 \mathrm{~d}^{-1}$ is marked. The two peaks at about 1.5 and $2.5 \mathrm{~d}^{-1}$ are one day aliases of $F$. Bottom panel: residual amplitude spectrum where $1 / 2 F$, $2 F$, and $3 F$ are located at lower amplitudes.

velocity measurement of $21.6 \mathrm{~km} \mathrm{~s}^{-1}$. Liu et al. (1991) reported a radial velocity of $24.3 \mathrm{~km} \mathrm{~s}^{-1}$ and $28.5 \mathrm{~km} \mathrm{~s}^{-1}$ for HD 47887 and HD 47777, respectively.

Binary interaction is extremely important in the evolution of massive stars (Langer 2012; Sana et al. 2012) and it is therefore important to determine whether HD 47887 and HD 47777 are binaries. For HD 47887 we additionally obtained one highresolution $(R \sim 60000)$ spectrum in January 2012 with the Robert G. Tull Coudé Spectrograph attached to the $2.7 \mathrm{~m}$ telescope of Mc Donald Observatory and five high-resolution $(R \sim$ $85000)$ spectra in September 2013 with the HERMES fiber-fed spectrograph (Raskin et al. 2011) at the $1.2 \mathrm{~m}$ Mercator telescope on La Palma. For HD 47777 we analysed more high-resolution ESPaDOnS spectra, obtained in December 2007, February 2010, and February 2013 in polarimetric mode, which will be presented in detail by Alecian et al. (in prep.) in the framework of the Magnetism in Massive Stars (MiMeS) project (Wade et al. 2012). For all spectra we derived $v_{r}$ by fitting a Gaussian to the Stokes $I$ LSD profile (see Sect. 3.3). The average $v_{r}$ value (and its standard deviation) obtained from all analysed spectra is listed in Table 3. For both stars we detected weak $\left(<5 \mathrm{~km} \mathrm{~s}^{-1}\right)$ radial velocity variations, typical of spotted stars.

All $v_{r}$ values obtained for the two stars are slightly higher than the average cluster $v_{r}$ of $17.68 \pm 2.26 \mathrm{~km} \mathrm{~s}^{-1}$, reported by Kharchenko et al. (2005) on the basis of 13 stars. In contrast, Liu et al. (1991) listed 21 radial velocity measurements for a total of 16 stars in the field of view of NGC 2264. Except for two stars, which are very likely non-cluster-members or close binaries, the stars observed by Liu et al. (1991) indicate a cluster average radial velocity of about $24 \mathrm{~km} \mathrm{~s}^{-1}$, which agrees well with our measurements.

Despite the possible small difference between the measured radial velocity and the cluster average, the position of the two stars in the sky (see Fig. 2) and the colour-magnitude diagram (compared with that of the other cluster stars), as well as their proper motion (Kharchenko et al. 2004), strongly suggest that both stars are members of the NGC 2264 open cluster.

\subsection{Fundamental parameters and abundance analysis}

We determined the projected rotational velocity $(v \sin i)$ using the Fourier transform method (Simón-Díaz \& Herrero 2007). The $v \sin i$ values, listed in Table 3, were derived on the basis of the Si III line at $\lambda \approx 4553 \AA$ and agree well with those reported in the literature by Balona (1975) and Vogel \& Kuhi (1981). We also searched for macroturbulence broadening, which we modelled as radial-tangential, but found it to be negligible, in agreement with the findings on macroturbulence broadening in massive stars reported by Simón-Díaz \& Herrero (2014).

Given the higher $\mathrm{S} / \mathrm{N}$, we determined the fundamental parameters and chemical abundances using the ESPaDOnS spectra of the two stars obtained in 2012, which were modelled using the FASTWIND (Fast Analysis of STellar atmospheres with WINDs; Santolaya-Rey et al. 1997; Puls et al. 2005) stellar atmosphere code. The code enables one to perform non-LTE calculations and assumes spherical symmetry with an explicit calculation of stellar winds by a $\beta$-like wind velocity law (Schaerer $\&$ Schmutz 1994). Because the terminal wind velocity cannot be constrained without high-resolution UV spectra (Puls et al. 2008), we adopted the values obtained from the empirical calibration given in Eq. (2) of Castro et al. (2012) for both stars.

We characterised the spectra on the basis of well-known transitions in the 4000-7000 ̊ wavelength range (see Crowther et al. 2006). We determined the effective temperature $\left(T_{\text {eff }}\right)$ and surface gravity $(\log g$ ) by simultaneously fitting the ionisation balance of He (i.e., He I-II) and Si (i.e., Si II-III-IV), and the shape of the He I-II and H I Balmer line profiles, searching for the combination of stellar parameters that best reproduced all features taken into account. The parameter determination was based on a large grid of FASTWIND stellar atmosphere models and on a set of routines designed to automatically find the best fit through a $\chi^{2}$ minimisation algorithm (see Lefever 2007). A description of the technique and stellar parameters covered by the adopted grid of stellar atmosphere models is provided by Castro et al. (2012). The determined atmospheric parameters for the two stars are listed in Table 3.

Geneva (Rufener et al. 1966) and Strömgren (Hauck \& Mermilliod 1997) photometry are available for HD 47887 and HD 47777, respectively. Adopting the calibration for the Geneva photometry given by Kunzli et al. (1997), for HD 47887 we obtained an effective temperature of $25700 \mathrm{~K}$ and a surface gravity of 4.25 , which agree, within the error bars, with the values we derived spectroscopically. Table 4 lists the fundamental parameters determined from Strömgren photometry for HD 47777, derived by adopting calibrations from Moon \& Dworetsky (1985), Balona (1994), Napiwotzki et al. (1993), and Castelli et al. (1997). To calibrate the photometry we adopted the TempLogG ${ }^{\text {TNG }}$ tool described by Kaiser (2006). The effective temperatures derived photometrically and spectroscopically agree well while for $\log g$ the photometric values are slightly higher than the spectroscopic ones. Note that Kaiser (2006) 
Table 3. Derived parameters and abundances for HD 47887 (second column) and HD 47777 (third column).

\begin{tabular}{|c|c|c|c|c|}
\hline & HD 47887 & HD 47777 & \multicolumn{2}{|r|}{ Sun } \\
\hline$T_{\text {eff }}[\mathrm{K}]$ & $24000 \pm 1000$ & $22000 \pm 1000$ & \multicolumn{2}{|c|}{5777} \\
\hline $\log g$ & $4.1 \pm 0.1$ & $4.2 \pm 0.1$ & \multicolumn{2}{|c|}{4.438} \\
\hline$v_{\text {mic }}\left[\mathrm{km} \mathrm{s}^{-1}\right]$ & $5 \pm 1$ & $4 \pm 1$ & \multicolumn{2}{|c|}{0.875} \\
\hline$v_{r}\left[\mathrm{~km} \mathrm{~s}^{-1}\right]$ & $22.9 \pm 1.5(7)$ & $24.9 \pm 1.3(11)$ & \multicolumn{2}{|c|}{-} \\
\hline$v \sin i\left[\mathrm{~km} \mathrm{~s}^{-1}\right]$ & 45 & 60 & \multicolumn{2}{|r|}{1.2} \\
\hline Rot. period [d] & 1.947807 & 2.640426 & \multicolumn{2}{|c|}{24.47} \\
\hline$v_{\mathrm{eq}}\left[\mathrm{km} \mathrm{s}^{-1}\right]$ & 116 & 67 & \multicolumn{2}{|c|}{2.067} \\
\hline$i\left[^{\circ}\right]$ & 23 & 64 & \multicolumn{2}{|c|}{$\begin{array}{l}2.001 \\
-\end{array}$} \\
\hline $\log L / L_{\odot}$ & $3.80 \pm 0.15$ & $3.42 \pm 0.15$ & \multicolumn{2}{|r|}{0.0} \\
\hline $\operatorname{age}_{\text {evol }}[\log t]$ & $7.00_{-0.18}^{+0.15}$ & $6.92_{-0.28}^{+0.28}$ & \multicolumn{2}{|r|}{9.66} \\
\hline$M_{\mathrm{evol}}\left[M_{\odot}\right]$ & $9.4_{-0.7}^{+0.6}$ & $7.6_{-0.5}^{+0.5}$ & \multicolumn{2}{|r|}{1.0} \\
\hline$R_{\mathrm{evol}}\left[R_{\odot}\right]$ & $4.4_{-0.4}^{+0.5}$ & $3.6_{-0.3}^{+0.4}$ & \multicolumn{2}{|r|}{1.0} \\
\hline $\begin{array}{l}R_{\mathrm{SED}}\left[R_{\odot}\right] \\
\left\langle B_{z}\right\rangle[\mathrm{G}]\end{array}$ & $\begin{array}{c}4.45 \pm 0.50 \\
373 \pm 48\end{array}$ & $\begin{array}{c}3.50 \pm 0.50 \\
469 \pm 87\end{array}$ & \multicolumn{2}{|r|}{1.0} \\
\hline & & & LTE & non-LTE \\
\hline He [dex] & $-1.04 \pm 0.10$ & $-1.74 \pm 0.10$ & -1.04 & -1.11 \\
\hline $\mathrm{C}[\mathrm{dex}]$ & $-4.35 \pm 0.10$ & $-4.15 \pm 0.13$ & -3.49 & -3.61 \\
\hline $\mathrm{N}[\operatorname{dex}]$ & $-4.36 \pm 0.10$ & $-4.36 \pm 0.13$ & -4.07 & -4.21 \\
\hline$O[\mathrm{dex}]$ & $-3.28 \pm 0.10$ & $-3.48 \pm 0.10$ & -3.17 & -3.35 \\
\hline $\mathrm{Ne}^{*}[\mathrm{dex}]$ & $-3.62 \pm 0.08(2)$ & $-3.89 \pm 0.05(2)$ & -3.96 & -4.11 \\
\hline $\operatorname{Mg}[\operatorname{dex}]$ & $-5.01 \pm 0.15$ & $-4.81 \pm 0.19$ & -4.46 & -4.44 \\
\hline $\mathrm{Al}^{*}[\mathrm{dex}]$ & $-6.26 \pm 0.08(3)$ & $-6.18 \pm 0.10(4)$ & -5.57 & -5.59 \\
\hline Si [dex] & $-4.83 \pm 0.10$ & $-4.83 \pm 0.20$ & -4.49 & -4.53 \\
\hline $\mathrm{P}^{*}[\mathrm{dex}]$ & & $-5.93(1)$ & -6.59 & -6.63 \\
\hline $\mathrm{S}^{*}[\mathrm{dex}]$ & $-5.05(1)$ & $-5.38 \pm 0.11(6)$ & -4.71 & -4.92 \\
\hline Ar* [dex] & $-5.39(1)$ & $-5.30(1)$ & -5.52 & -5.64 \\
\hline $\mathrm{Fe}^{*}[\mathrm{dex}]$ & $-4.52 \pm 0.32(14)$ & $-4.82 \pm 0.11(7)$ & -4.54 & -4.54 \\
\hline
\end{tabular}

Notes. The listed radial velocity $v_{r}$ is the average value obtained from all spectra analysed in this work, and the number in parenthesis indicates the number of spectra for which we determined a $v_{r}$ value. The angle $i$, in degrees, corresponds to the inclination angle of the star's rotational axis to the line of sight. $R_{\text {evol }}$ and $R_{\mathrm{SED}}$ indicate the stellar radius obtained from the comparison to the evolutionary tracks (see Sect. 4) and from the fitting of the synthetic fluxes to the observed photometry converted to physical units (see Sect. 3.4). For comparison, the LTE and non-LTE solar abundance values obtained by Grevesse et al. (1996) and Asplund et al. (2009), respectively, are given in the fourth and fifth columns. The abundances, listed in the second half of the table, are given in $\log \left(N / N_{\text {tot }}\right)$ and the number in parenthesis indicates the number of lines used to determine the element abundance. The abundance of the elements marked with an asterisk was determined assuming LTE on the basis of the final FASTWIND models, as described in Sect. 3.2.

Table 4. Comparison between photometric and spectroscopic atmospheric parameters.

\begin{tabular}{lcc}
\hline \hline Calibration & $\begin{array}{c}T_{\text {eff }} \\
{[\mathrm{K}]}\end{array}$ & $\log g$ \\
\hline Moon \& Dworetsky (1985) & 22254 & 4.30 \\
Balona (1994) & 21383 & 4.40 \\
Napiwotzki et al. (1993) & 21847 & 4.55 \\
Castelli et al. (1997) & 20951 & 4.67 \\
\hline This work & 22000 & 4.2 \\
& \pm 1000 & \pm 0.1 \\
\hline
\end{tabular}

Notes. Effective temperature and surface gravity derived from Strömgren photometry (Hauck \& Mermilliod 1997) for HD 47777 and using calibrations from Moon \& Dworetsky (1985), Balona (1994), Napiwotzki et al. (1993), and Castelli et al. (1997). The last two lines list the $T_{\text {eff }}$ and $\log g$ values derived from the ESPaDOnS spectrum in this work.

concluded that Moon \& Dworetsky (1985) provided the most reliable calibration of Strömgren photometry for B-type mainsequence stars. In addition, we also note that the $T_{\text {eff }}$ value we derived for HD 47777 agrees well with that reported by Glagolevskij (1994).

We determined the microturbulence velocity $\left(v_{\text {mic }}\right)$ and nonLTE abundances of $\mathrm{C}, \mathrm{N}, \mathrm{O}, \mathrm{Si}$, and $\mathrm{Mg}$ by adopting the set of best-fitting fundamental parameters and using FASTWIND models computed by varying $v_{\text {mic }}$ in steps of $1 \mathrm{~km} \mathrm{~s}^{-1}$ and the abundance of each considered element in steps of 0.2 dex. A detailed description of the lines included in the non-LTE analysis and of the adopted model atoms in the 3900-5000 $\AA$ wavelength range is provided by Castro et al. (2012). Note that the technique has been substantially updated since Castro et al. (2012) as follows: the chemical analysis was handled almost in a fully automatic way using a genetic algorithm approach for finding the best composition of models fitting the analysed spectral lines. The $v_{\text {mic }}$ and abundance values derived from the non-LTE analysis are listed in Table 3.

To derive the abundance of the other elements presenting measurable features in the spectra of the two stars, we used the final adopted FASTWIND models to fit synthetic spectra, calculated with SYNTH3 (Kochukhov 2007), to the observations. For the spectral synthesis calculation with SYNTH3, we adopted the Vienna Atomic Line Database (VALD; Piskunov et al. 1995; Kupka et al. 1999; Ryabchikova et al. 1999) as source for the atomic line parameters. The derived LTE abundances are listed in Table 3 and are marked with an asterisk (*). Because SYNTH3 assumes LTE, the abundance of the elements obtained in this way has to be taken with caution. The uncertainty on the LTE abundances is the standard deviation from the average abundance and does not account for the uncertainties on the 
Table 5. Results from the LSD analysis.

\begin{tabular}{|c|c|c|c|c|c|c|c|}
\hline $\begin{array}{l}\text { Star } \\
\text { name }\end{array}$ & $\begin{array}{c}\left\langle B_{z}\right\rangle(V) \\
{[\mathrm{G}]} \\
\end{array}$ & $\begin{array}{c}\text { FAP }(V) \\
\text { line }\end{array}$ & $\begin{array}{c}\text { Detection } \\
V \\
\end{array}$ & $\begin{array}{c}\text { FAP }(V) \\
\text { blue cont./red cont. }\end{array}$ & $\begin{array}{l}S / N \\
I_{\mathrm{LSD}} \\
\end{array}$ & $\begin{array}{l}S / N \\
V_{\mathrm{LSD}} \\
\end{array}$ & \# lines \\
\hline HD 47887 & $373 \pm 48$ & $<10^{-15}$ & DD & $7.8 \times 10^{-1} / 7.3 \times 10^{-1}$ & 820 & 5652 & 256 \\
\hline HD 47777 & $469 \pm 87$ & $2.8 \times 10^{-12}$ & DD & $4.7 \times 10^{-1} / 9.2 \times 10^{-1}$ & 1247 & 7734 & 141 \\
\hline Star & $\left\langle B_{z}\right\rangle(N)$ & FAP $(N)$ & Detection & $\operatorname{FAP}(N)$ & $S / N$ & $S / N$ & \# lines \\
\hline Name & {$[\mathrm{G}]$} & line & $N$ & blue cont./red cont. & $I_{\mathrm{LSD}}$ & $V_{\mathrm{LSD}}$ & \\
\hline HD 47887 & $4 \pm 46$ & $7.7 \times 10^{-1}$ & ND & $4.0 \times 10^{-1} / 9.7 \times 10^{-1}$ & 820 & 5652 & 256 \\
\hline HD 47777 & $11 \pm 86$ & $2.5 \times 10^{-1}$ & ND & $8.6 \times 10^{-1} / 2.2 \times 10^{-2}$ & 1247 & 7734 & 141 \\
\hline
\end{tabular}

Notes. The S/N Stokes $I$ and $V$ is that of the LSD profile. Columns two and three list the average longitudinal magnetic field $\left(\left\langle B_{z}\right\rangle\right)$ and the FAP for both Stokes $V$ and the $N$ profile. Column four expresses a magnetic field detection on the basis of the FAP, where DD indicates a definite detection (FAP $\left.<10^{-5}\right)$, MD indicates a marginal detection $\left(10^{-5}<\right.$ FAP $\left.<10^{-3}\right)$, and ND incicates a non-detection (FAP $\left.>10^{-3}\right)$. Column five lists the FAP calculated in the continuum region bluewards and redwards of the spectral line, over a range as broad as that adopted to derive $\left\langle B_{z}\right\rangle$. The last column lists the number of lines used in the line mask.

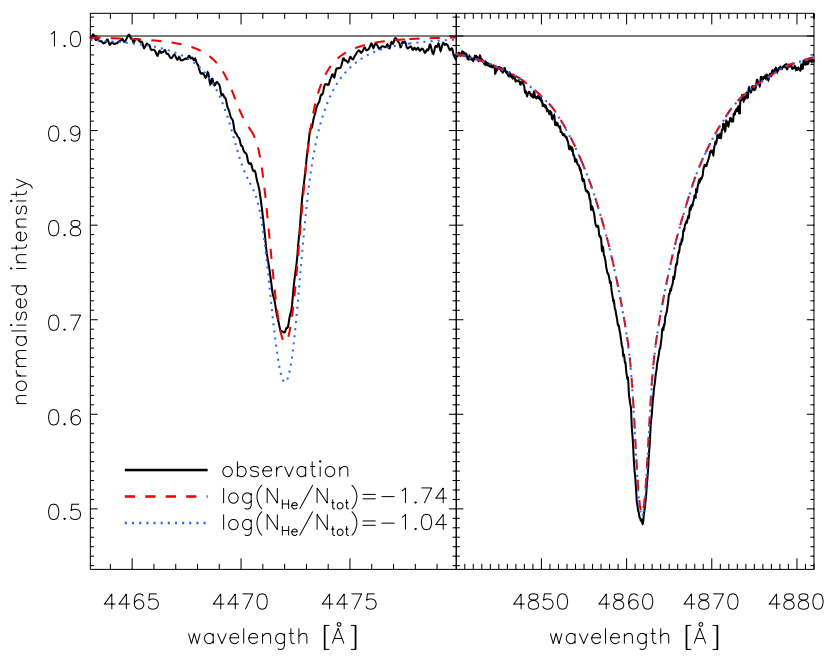

Fig. 7. Left panel: comparison between the observed He I line profile at $\sim 4471$ A (black solid line) of HD 47777 and non-LTE synthetic spectra calculated with the solar (blue dotted line) and adopted (red dashed line) He abundance. For both synthetic spectra we adopted the final $T_{\text {eff }}$ value of 22000 K. Right panel: as for the left panel, but for the $\mathrm{H} \beta$ line.

stellar parameters. Following Fossati et al. (2009), the contribution of the uncertainties on $\log g$ and $v_{\text {mic }}$ on the abundance uncertainties is almost negligible, while, depending on the element, the uncertainty on $T_{\text {eff }}$ probably adds another $0.1-0.2$ dex to the abundance uncertainties listed in Table 3.

We obtained a solar He abundance for HD 47887, while HD 47777 is a He-weak star. The left panel of Fig. 7 shows the observed HD 47777 He I line profile at $\sim 4471 \AA$ in comparison with non-LTE synthetic spectra calculated with the solar and adopted $\mathrm{He}$ abundance. When fitting the $\mathrm{He}$ I lines with the solar He abundance, we obtained an effective temperature of $16000 \mathrm{~K}$ and a surface gravity $\log g$ of 3.4, which disagrees with the photometric parameters and also leads to a considerably poorer fit of the Si II/Si III ionisation equilibrium, even when the microturbulence velocity is tuned. Because of the He underabundance, we determined the stellar parameters using only the hydrogen Balmer lines and the Si II/Si III ionisation equilibrium.

For a more consistent comparison, Table 3 lists the LTE and non-LTE solar abundances determined by Grevesse et al. (1996) and Asplund et al. (2009). For HD 47887, within the uncertainties most of the abundances derived accounting for non-LTE are similar to those of the Sun, except for $\mathrm{C}, \mathrm{Mg}$, and $\mathrm{Si}$, which are underabundant. For the elements analysed assuming LTE,
non-LTE corrections for early B-type stars are available only for Ne (see e.g., Hempel \& Holweger 2003; Cunha et al. 2006; Morel \& Butler 2008; Przybilla et al. 2008). Cunha et al. (2006) showed that at the temperature of HD 47887, the Ne non-LTE correction is about $-0.5 \mathrm{dex}$, bringing our $\mathrm{Ne}$ abundance into agreement with the non-LTE solar abundance value.

For the elements analysed to account for non-LTE effects, the abundance pattern we obtained for HD 47777 is remarkably similar $(\mathrm{C}, \mathrm{Mg}$, and $\mathrm{Si}$ are underabundant, while $\mathrm{N}$ and $\mathrm{O}$ are solar) to that of HD 47887, except for a He underabundance of HD 47777. Assuming the non-LTE correction given by Cunha et al. (2006), we obtained a Ne underabundance for HD 47777. For this star, the phosphorus abundance was determined on the basis of just one PI line for which the oscillator strength was taken from calculations by Hibbert (1988). Therefore, at least part of the observed overabundance may be caused by uncertainties in the calculated transition probability.

\subsection{High-precision magnetic field detection}

To detect magnetic fields we used LSD (Donati et al. 1997), which combines line profiles centred at the position of the individual lines and scaled according to the line strength, wavelength, and sensitivity to a magnetic field. The resulting mean profiles $(I, V$, and $N$ ) were obtained by combining about 200 spectral lines with a strong increase in S/N and therefore sensitivity to polarisation signatures. We computed the LSD profiles of Stokes $I, V$, and of the null profile (for a definition of null profile see Bagnulo et al. 2009) using the methodology and the code described by Kochukhov et al. (2010). We prepared the line mask used by the LSD code separately for the two stars, adopting the stellar parameters and abundances listed in Table 3. We extracted the line parameters from VALD. We used all available metallic and He lines stronger than $10 \%$ of the continuum for the two stars, avoiding hydrogen lines and removing the spectral regions affected by telluric lines. The velocity range taken into account to calculate the average longitudinal magnetic field $\left(\left\langle B_{z}\right\rangle\right.$; see also Borra et al. 1984) is marked in Fig. 8.

Figure 8 shows the obtained LSD profiles, while Table 5 gives the results gathered from their analysis. We defined the magnetic field detection making use of the false-alarm probability (FAP): following Donati et al. (1992), we considered a profile with an FAP $<10^{-5}$ as a definite detection (DD), $10^{-5}<$ FAP $<$ $10^{-3}$ as a marginal detection (MD), and an FAP $>10^{-3}$ as a non-detection (ND). To verify that the magnetic field detections are not spurious, we calculated the FAP for the null profile in the same velocity range as used for the magnetic field, obtaining 

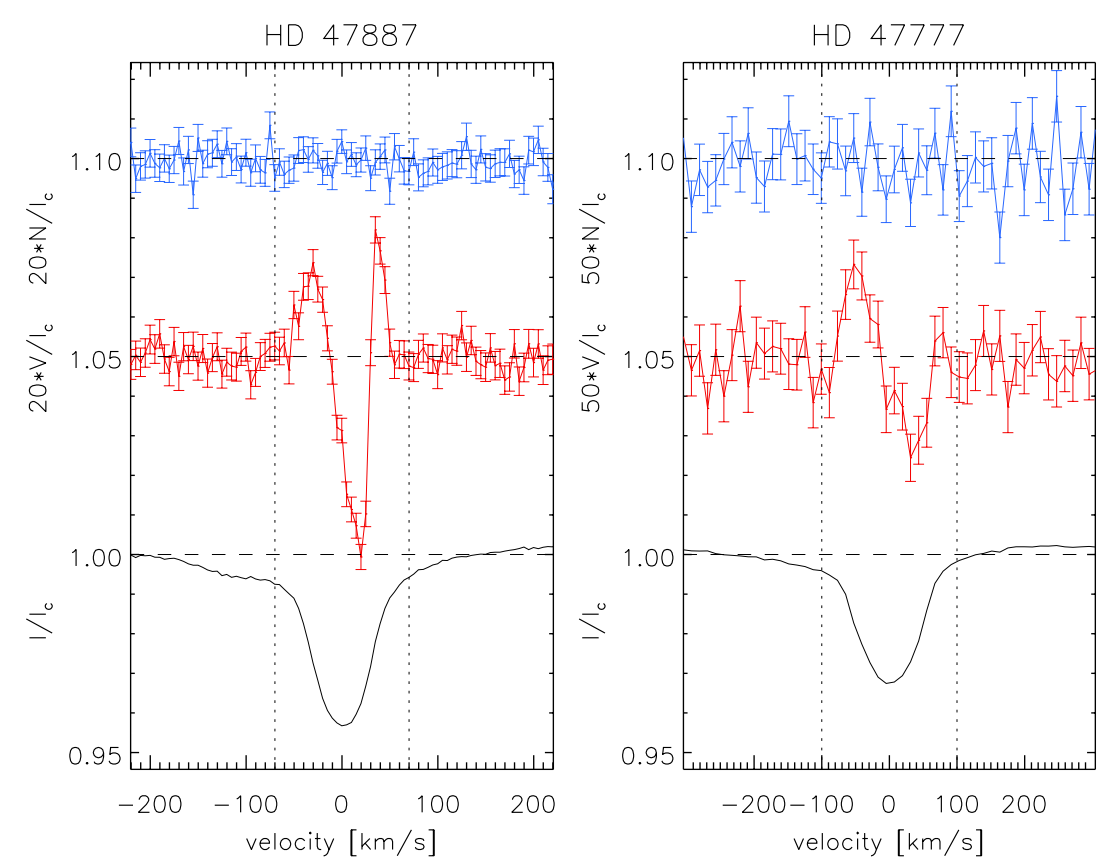

Fig. 8. $I, V$, and $N$ LSD profiles (from bottom to top) obtained for HD 47887 (left panel) and HD 47777 (right panel). The $V$ and $N$ profiles were expanded by a factor of 20 for HD 47887 and 50 for HD 47777, and were shifted upwards for visualisation purposes. All profiles were shifted to the rest frame. The vertical dotted lines indicate the ranges adopted to calculate the magnetic field. an ND in both cases. We also calculated the FAP for equivalent velocity ranges displaced both redwards and bluewards to sample the continuum in the Stokes $I$ spectrum. The results for both Stokes $V$ and the null profile are given in Col. 5 of Table 5. The FAP obtained in these tests are very close to 1.0, allowing us to conclude that our detections are most likely genuine. In addition, we verified that both Stokes $V$ and the null profile are consistent with the expected noise properties (e.g., Stokes $V$ uncertainties consistent with the standard deviation of the null profile; zero-order moment of Stokes $V$ consistent with zero). Finally, even in rather extreme cases (de la Chevrotière et al. 2013), crosstalk between linear and circular polarisation would not be able to lead to the observed Stokes $V$ signatures, because it would require a strong linear polarisation of the photospheric spectral lines, which is not expected for main-sequence early B-type stars. Moreover, the measured crosstalk of ESPaDOnS (performed periodically since 2010) is negligible ${ }^{1}$.

For HD 47887 the double-reversal shape of the Stokes $V$ LSD profile suggests that the observed stellar surface presents two magnetic poles. On the other hand, for HD 47777 the shape of the Stokes $V$ LSD profile indicates that the observed stellar surface presents one magnetic pole.

\subsection{Spectral energy distribution}

To examine the quality of the derived atmospheric parameters in more detail and determine the radii of the two stars we compared the synthetic fluxes, calculated with FASTWIND, with fluxcalibrated photometry. Figures 9 and 10 show the comparison of the FASTWIND synthetic fluxes, calculated by adopting the fundamental parameters (and abundances) derived for HD 47887 and HD 47777 with the available ultraviolet (UV), optical, and infrared (IR) photometry. We show the Johnson (Mermilliod 1991), 2MASS (Zacharias et al. 2005), Spitzer (IRAC Sung et al. 2009), and WISE (Cutri et al. 2012) photometry, converted to physical units for the two stars. For the conversion we used the calibrations provided by Bessel et al. (1998), van der Bliek et al. (1996), Sung et al. (2009), and Wright et al. (2010). For

\footnotetext{
1 http://www.cfht.hawaii.edu/Instruments/Spectroscopy/ Espadons/
}

HD 47777 we also show the available TD1 (Thompson et al. 1978) photometry and the Spitzer MIPS photometric value at $24 \mu \mathrm{m}$ from Sung et al. (2009), adopting the calibration given by the Spitzer MIPS Instrument handbook ${ }^{2}$.

Because FASTWIND was not conceived for carrying out analyses in the UV domain and the region across the Balmer jump was calculated without accounting for the high order Balmer lines, we used the LLMODELS stellar model atmosphere code (Shulyak et al. 2004) for the two stars to compute LTE synthetic fluxes from the far-UV to the far-infrared wavelength region. LLMODELS assumes LTE and plane-parallel geometry and takes into account the individualised abundance pattern for opacity calculations. The LLMODELS synthetic fluxes are also shown in Figs. 9 and 10.

For the fit performed to determine the stellar radii, we took into account the FASTWIND synthetic fluxes and the TD1 (for HD 47777), Johnson, and 2MASS photometry (note that we did not consider the TD1 flux value at $\sim 2800 \AA$, which is affected by systematics; see e.g., Morel et al. 2014). We excluded the far-IR photometry from the fit to highlight infrared excess. The adopted cluster distance $(743 \pm 80 \mathrm{pc})$ and reddening $(E(B-V)=$ $0.061 \pm 0.011 \mathrm{mag})$ were derived by averaging the results obtained by various photometric studies of this cluster, that is, Gray (1965), Spassova \& Beav (1985), Lynga (1985), Battinelli et al. (1994), Malysheva (1997), Sung et al. (1997), Dambis (1999), Loktin (2001), Lata (2002), and Kharchenko et al. (2005). For HD 47887 we derived a stellar radius of $4.45 \pm 0.50 R_{\odot}$, while for HD 47777 we derived a radius of $3.50 \pm 0.50 R_{\odot}$. Note also the infrared excess shown by the WISE and MIPS photometry, which might be due to a problem in the background subtraction. We corrected the fluxes for the interstellar reddening using the parametrisation by Fitzpatrick (1999) and assuming a total-toselective extinction $R(V)$ of 3.1 .

Figure 11 shows a comparison between the FASTWIND and LLMODELS synthetic fluxes of HD 47777 and the available far-UV FUSE spectrum, which we retrieved (reduced and

\footnotetext{
2 http://irsa.ipac.caltech.edu/data/SPITZER/docs/mips/ mipsinstrumenthandbook/
} 

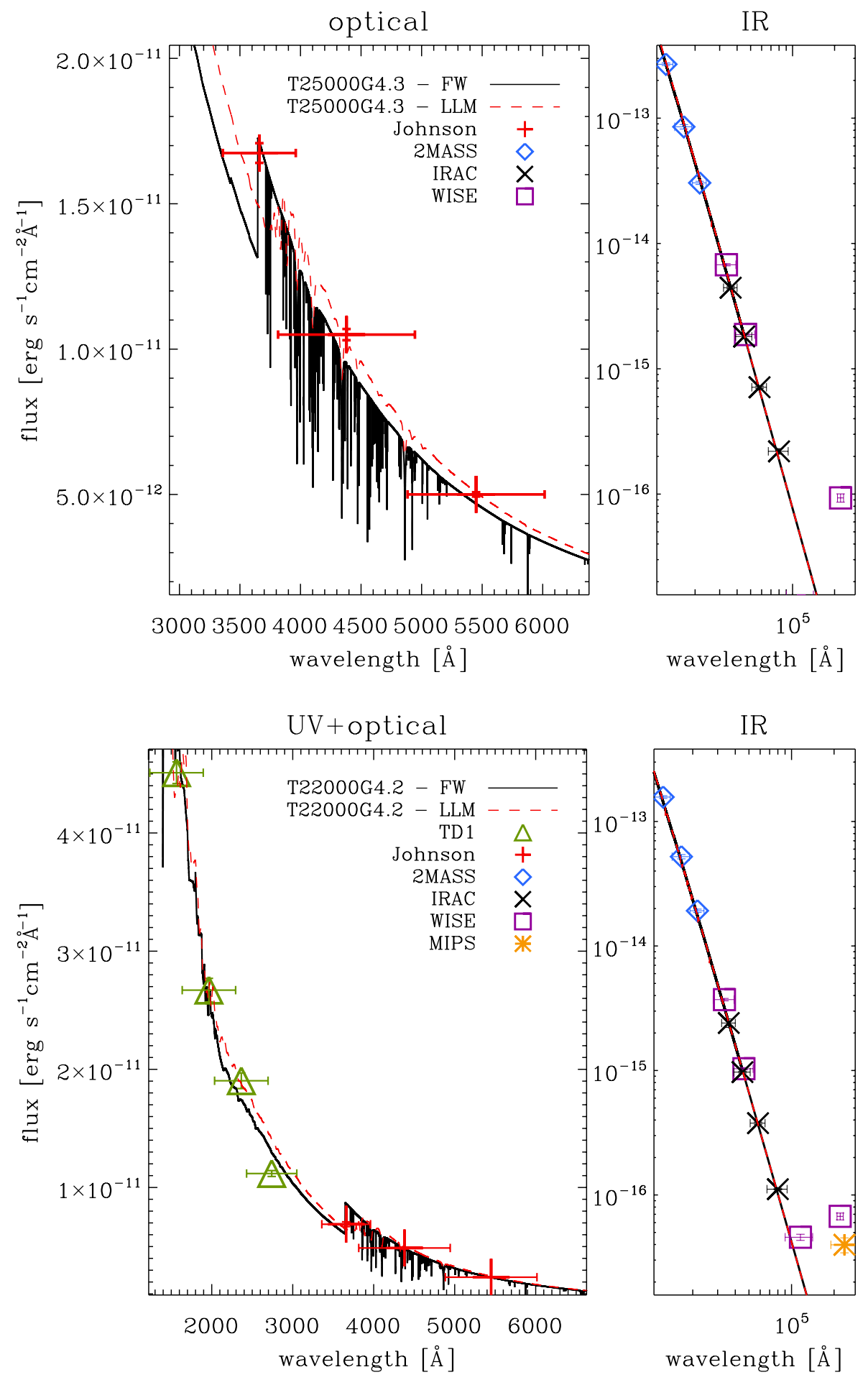

Fig. 9. Comparison between FASTWIND (full black line) and LLMODELS (dashed red line) theoretical fluxes, calculated with the fundamental parameters derived for HD 47887, with Johnson (red pluses), 2MASS (blue diamonds), Spitzer-IRAC (black crosses), and WISE (purple squares) photometry converted into physical units. The optical and IR spectral regions are shown in the right and left panel, respectively. The synthetic fluxes take into account the cluster distance and reddening given in the text and a stellar radius of $4.45 R_{\odot}$. The horizontal bars show the full width at half maximum of the photometric filters.
Fig. 10. Comparison between FASTWIND (full black line) and LLMODELS (dashed red line) theoretical fluxes, calculated with the fundamental parameters derived for HD 47777, with TD1 (green triangles), Johnson (red pluses), 2MASS (blue diamonds), Spitzer-IRAC (black crosses), WISE (purple squares), and SpitzerMIPS (yellow asterisk) photometry converted into physical units. The UV and optical spectral regions are shown in the left panel, while the right panel shows the IR band. The synthetic fluxes take into account the cluster distance and reddening given in the text and a stellar radius of $3.50 R_{\odot}$. The horizontal bars show the full width at half maximum of the photometric filters. calibrated) from the MAST archive ${ }^{3}$. The synthetic fluxes shown in Fig. 11 account for the cluster distance and reddening given above, plus the derived stellar radius of $3.5 R_{\odot}$. We did not include the FUSE spectrum in the fit of the spectral energy distribution, because it allows one to check the adopted interstellar reddening. Note that in contrast to the other works in which we analysed members of the NGC 2264 open cluster (Zwintz et al. 2013a,b), we decided to adopt a different cluster distance and reddening. Our decision was based on the fact that adopting the reddening of $E(B-V)=0.071 \mathrm{mag}$, given by Sung et al. (1997)

\footnotetext{
3 http://archive.stsci.edu/
}

and used by Zwintz et al. (2013a,b), we were not able to fit the observed far-UV fluxes, which are instead well reproduced with the lower adopted value of $E(B-V)=0.061 \mathrm{mag}$. Because the stars belong to a young open cluster, we cannot exclude that the resulting difference in reddening is caused by deviations from the used extinction law, which would be particularly noticeable in the far-UV.

From the rotational periods derived from the MOST light curves (Sect. 2), the $v \sin i$ values derived from the highresolution spectra (Sect. 3.2), and the stellar radii derived from the fit of the spectral energy distribution, we determined the equatorial rotational velocity and the stellar inclination angle. 


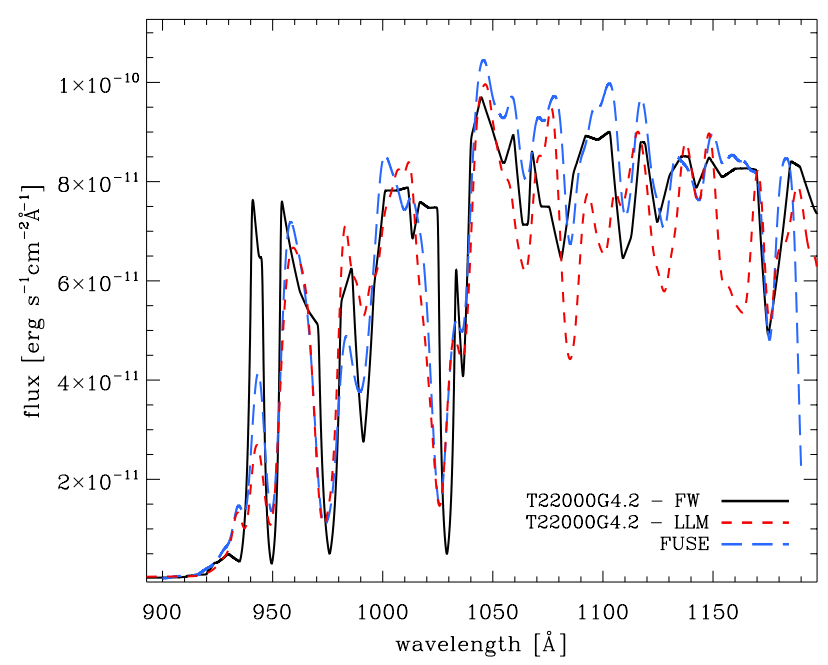

Fig. 11. Comparison between FASTWIND (full black line) and LLMODELS (dashed red line) theoretical fluxes, calculated with the fundamental parameters derived for HD 47777, with the available fluxcalibrated FUSE spectrum (blue long-dashed line). The synthetic fluxes take into account the cluster distance and reddening given in the text and a stellar radius of $3.50 R_{\odot}$.

For HD 47887 we obtained an equatorial rotational velocity $\left(v_{\text {eq }}\right)$ of $116 \mathrm{~km} \mathrm{~s}^{-1}$, leading to an inclination angle of $23^{\circ}$ (the angle between the star's rotation axis and the line of sight, $i$ ). For HD 47777 we derived an equatorial rotational velocity of $67 \mathrm{~km} \mathrm{~s}^{-1}$ and an inclination angle of $64^{\circ}$. Our results show that HD 47887 is viewed close to the rotational pole, while HD 47777 can be considered as viewed almost equator-on. As a consequence, assuming a dipolar magnetic field configuration, the fact that the Stokes $V$ LSD profile of HD 47887 suggests we observed two poles of the magnetic field implies that the rotation and magnetic axis have different inclination angles, as found for most magnetic stars. Nevertheless, it is possible that HD 47887 has a complex magnetic field geometry, as shown by other magnetic early B-type stars, such as $\tau$ Sco (Donati et al. 2006), HD 37776 (Kochukhov et al. 2011).

\subsection{Hertzsprung-Russell diagram}

Figure 12 shows the position of HD 47887 and HD 47777 in the Hertzsprung-Russell (HR) diagram in comparison with Milky Way composition evolutionary tracks for $7 M_{\odot}, 9 M_{\odot}$, and $10 M_{\odot}$ stars (Brott et al. 2011). We calculated the luminosities of the two stars on the basis of the Johnson $V$-band magnitude (Walker 1956) and of the distance and reddening adopted in Sect. 3.4. We used the bolometric correction provided by the FASTWIND model, which agrees well with that obtained with the calibration given by Balona (1994). Luminosities are listed in Table 3 and their uncertainties take into account the uncertainty in the distance and the bolometric correction, the latter assumed to be $0.1 \mathrm{mag}$.

We determined the masses, radii, and ages of the two stars using the BONNSAI code (Schneider et al. 2013a; Schneider et al., in prep.) and the Milky Way stellar evolution models of Brott et al. (2011). BONNSAI computes the posterior probability distribution, $p(\mathbf{m} \mid \mathbf{d})$, of stellar model parameters, $\mathbf{m}$, given observational data, d, using Bayes' theorem:

$p(\mathbf{m} \mid \mathbf{d}) \propto p(\mathbf{d} \mid \mathbf{m}) p(\mathbf{m})$,

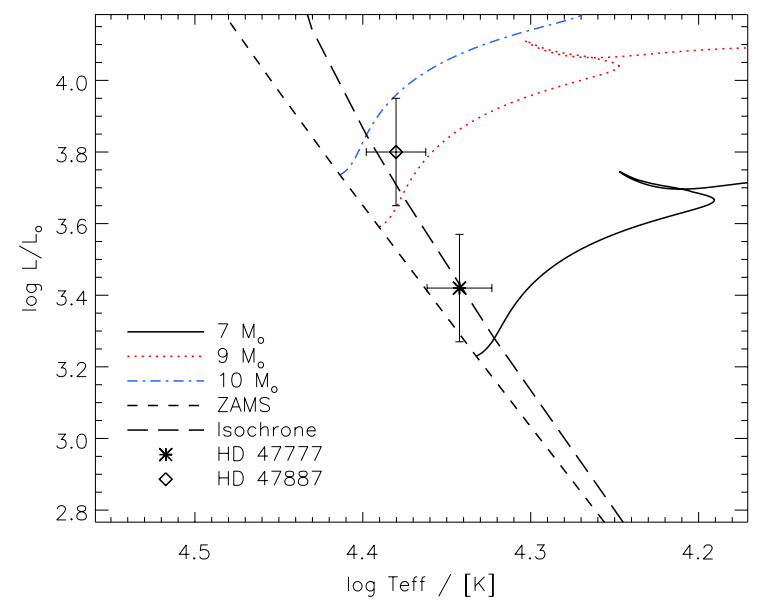

Fig. 12. Position of HD 47887 (diamond) and HD 47777 (asterisk) in the Hertzsprung-Russell (HR) diagram in comparison with Milky Way composition evolutionary tracks for $7 M_{\odot}$ (black solid line), $9 M_{\odot}$ (red dotted line), and $10 M_{\odot}$ (blue dash-dotted line) stars computed by Brott et al. (2011). The black short-dashed line shows the zero-age main sequence (ZAMS), while the black long-dashed line shows the isochrone calculated with the adopted cluster age of $\log t=6.89$.

where $p(\mathbf{d} \mid \mathbf{m})$ is the likelihood and $p(\mathbf{m})$ the prior. We adopted a Gaussian likelihood, used a standard Salpeter initial mass function (Salpeter 1955) as prior of the initial mass as well as flat priors for the two remaining stellar model parameters, stellar age, and initial equatorial rotational velocity (the initial chemical composition of stars is not a free parameter because we used stellar models with a fixed chemical composition). Adopting flat priors means that all stellar ages and initial equatorial rotational velocities are a priori equally probable. The advantages of this method for our application are that (i) all available observables are simultaneously matched to stellar models, taking the observed uncertainties and a priori knowledge into account; and (ii) robust uncertainties for the stellar parameters are derived from the posterior probability distribution.

We simultaneously match the observational data $\mathbf{d}=$ $\left(\log L / L_{\odot}, T_{\text {eff }}, \log g, v_{\text {eq }}\right.$ ) (see Table 3) to stellar models to compute the posterior probability distribution of the stellar parameters. We adopted $1 \sigma$ uncertainties of $0.15 \mathrm{dex}$ on $\log L / L_{\odot}$, $1000 \mathrm{~K}$ on $T_{\text {eff }}, 0.1 \mathrm{dex}$ on $\log g$ and $20 \mathrm{~km} \mathrm{~s}^{-1}$ on $v_{\text {eq. }}$. The stellar models reproduce the set of observables well, that is, each observable is not farther away from the best-matching stellar model than $\sim 0.2 \sigma$.

For HD 47887 and HD 47777 we obtained initial stellar masses of $9.4_{-0.7}^{+0.6} M_{\odot}$ and $7.6_{-0.5}^{+0.5} M_{\odot}$, and stellar radii of $4.4_{-0.4}^{+0.5} R_{\odot}$ and $3.6_{-0.3}^{+0.4} R_{\odot}$, respectively. The most likely initial equatorial rotational velocities are $120_{-23}^{+19} \mathrm{~km} \mathrm{~s}^{-1}$ and $70_{-24}^{+19} \mathrm{~km} \mathrm{~s}^{-1}$, respectively. The given uncertainties are $1 \sigma$ confidence intervals. Mass loss can be considered to be negligible because we obtained a mass loss of about $3 \times 10^{-10} M_{\odot} / \mathrm{yr}$ for both stars.

The stellar radii obtained using the evolutionary tracks agree well with those we derived from the SED analysis (see Sect. 3.4). Note that the use of the stellar radii obtained from the evolutionary tracks changes the equatorial rotational velocity by only about $2 \mathrm{~km} \mathrm{~s}^{-1}$ for both stars. Hohle et al. (2010) used evolutionary tracks from three different authors to determine the mass of HD 47777. They obtained an average value of $6.31 \pm 3.54 M_{\odot}$, which, given the very large uncertainty, agrees with our estimated mass within one sigma. Nevertheless, the slightly lower 
value is most likely due to the lower effective temperature of $18700 \mathrm{~K}$ adopted by Hohle et al. (2010), which was based upon the B3V spectral classification.

We also determined the age for the two stars, obtaining $\log t=7.00_{-0.18}^{+0.15}$ and $\log t=6.92_{-0.28}^{+0.28}$ for HD 47887 and HD 47777, respectively. These values agree well with the cluster age of $\log t=6.89 \pm 0.26$, which we derived in the same way (i.e., averaging) as for the cluster distance and reddening (see Sect. 3.4). This agreement is also evident in the positions of the two stars on the HR diagram with an isochrone calculated for the adopted cluster age (see Fig. 12).

\section{Discussion and conclusions}

We have derived the physical properties of HD 47887 and HD 47777. The two stars are found to be core-hydrogen burning stars with evolutionary masses of $9.4 M_{\odot}$ and $7.6 M_{\odot}$ and possess strong magnetic fields of several hundred Gauss. Our photometric results imply that both stars show brightness inhomogeneities at their surfaces, which allowed us to derive their rotation periods of $1.9 \mathrm{~d}$ and $2.6 \mathrm{~d}$, with high accuracy. We conclude that both stars are rather slow rotators, with equatorial rotational velocities of $116 \mathrm{~km} \mathrm{~s}^{-1}$ and $67 \mathrm{~km} \mathrm{~s}^{-1}$.

Despite their similarity, there are distinct differences between the two stars. Our results are consistent with a simple, dipolar magnetic field structure for HD 47777, while HD 47887 appears to show two magnetic poles at its visible surface, possibly suggesting a more complex magnetic field geometry, perhaps similar to $\tau$ Sco (Donati et al. 2006). Furthermore, our atmosphere analysis indicates that the surface abundances of HD 47887 and HD 47777 are rather similar, but HD 47777 shows a strong He underabundance. Because the two stars are members of the same cluster, such a difference is probably not caused by different initial conditions, but by evolutionary processes. Because the low helium abundance of He-weak chemically peculiar stars is understood to be an effect of diffusion in the stellar envelope (see e.g., Michaud 1970; Michaud et al. 1979), our results imply that this effect can occur in stars of $\sim 7.6 M_{\odot}$, but not at the slightly higher mass of $9.4 M_{\odot}$. This could either be due to stronger subsurface convection at higher mass (Cantiello et al. 2009), or a strengthening of stellar-wind-induced mass loss (cf., Vick et al. 2010). (Note that the slightly higher equatorial rotational velocity and weaker magnetic field of the more massive star might also play a role.) In either case, the two stars analysed here are ideal candidates to test these conjectures.

The two stars we analysed may also shed light on the question of the origin of magnetic fields. One variant of the fossil-field hypothesis proposes that the magnetic fields in intermediate-mass and massive main-sequence stars are surviving stable remnants of the interstellar magnetic fields that thread the molecular clouds from which the stars formed (Donati \& Landstreet 2009). Since then the field has no other consequences than lowering the angular momentum of the forming stars, none of the derived properties of the two stars contradicts a fossil origin of the magnetic field.

Alternatively, the magnetic fields of the two stars could have been produced during a strong binary interaction, for instance, by a stellar merger (Langer 2013). While a merger product is expected to rotate rapidly at first (de Mink et al. 2013), a strong magnetic field generated during this process may induce substantial angular momentum loss and consequently slow rotation (Langer 2012). Furthermore, Glebbeek et al. (2013) predicted that while massive merger stars in general often show a surface nitrogen enrichment, their results also imply that in the mass range considered here merger stars may retain their abundances unaltered. Consequently, while the observed spin and surface abundances of HD 47887 and HD 47777 do not clearly support the binary hypothesis, neither do they dispute it.

A merging during main-sequence evolution leads to a rejuvenation of the merger product and transforms it into a blue straggler, whereas a pre-main-sequence merger caused by tidal effects from circumbinary material (Korntreff et al. 2012) would lead to a main-sequence star of normal age. Blue stragglers would naturally populate the high-mass end of the mass distribution of cluster stars (Schneider et al. 2013b). While Figs 1 and 12 show no clear evidence for HD 47887 and HD 47777 to be blue straggler stars, the upper main-sequence of NGC 2264 is populated only very sparsely. The fact that at least two of the ten brightest stars of NGC 2264 are magnetic, whereas the incidence of magnetic fields in early B-type stars in general is about 7\%, might be interpreted as support of the binary hypothesis.

We conclude that it is highly worthwhile to investigate the remaining massive stars in the NGC 2264 open cluster in some detail. Their stellar parameters may allow us to determine the age of this cluster more accurately, and to identify the blue straggler nature of any of the cluster stars. On the other hand, any detection of a magnetic field in the remaining about ten most massive cluster members would be spectacular, because it would render NGC 2264 the cluster with the highest concentration of magnetic main-sequence massive stars, together with the Orion Nebula Cluster, where three out of nine main-sequence massive stars are magnetic.

Acknowledgements. The research leading to these results has received funding from the European Research Council under the European Community's Seventh Framework Programme (FP7/2007-2013)/ERC grant agreement No. 227224 (PROSPERITY). This research is (partially) funded by the Research Council of the K.U. Leuven under grant agreement GOA/2013/012. K.Z. received a Pegasus Marie Curie Fellowship of the Research Foundation Flanders (FWO) during part of this work. G.A.W. acknowledges support from the Natural Sciences and Engineering Research Council of Canada (NSERC). A.A.T. is a Chercheur Qualifié FNRS. Spectroscopic data were obtained with the $2.7 \mathrm{~m}$ telescope at Mc Donald Observatory, Texas, US. Based on observations made with the Mercator Telescope, operated on the island of La Palma by the Flemish Community, at the Spanish Observatorio del Roque de los Muchachos of the Instituto de Astrofísica de Canarias. Based on observations obtained with the HERMES spectrograph, which is supported by the Funds for Scientific Research of Flanders (FWO), Belgium, the Research Council of K.U. Leuven, Belgium, the Fonds National Recherches Scientific (FNRS), Belgium, the Royal Observatory of Belgium, the Observatoire de Genève, Switzerland and the Thüringer Landessternwarte Tautenburg, Germany. Use was made of the WEBDA database of open clusters developed and maintained by E. Paunzen, C. Stütz and J. Janik at the Department of Theoretical Physics and Astrophysics of the Masaryk University, Brno, Czech Republic. We would also like to thank P. Beck and P. Degroote for fruitful discussions, A. Moffat for useful comments on the manuscript, and E. Paunzen and I. Müller for their support in the preparation of this work.

\section{References}

Abt, H. A. 1970, ApJS, 19, 387

Ahumada, J., \& Lapasset, E. 1995, A\&AS, 109, 375

Alecian, E., Catala, C., Wade, G. A., et al. 2009, Magnetism in Herbig Ae/Be stars and the link to the Ap/Bp stars, eds. C. Neiner, \& J.-P. Zahn, EAS Pub. Ser., 39, 121

Asplund, M., Grevesse, N., Sauval, A. J., \& Scott, P. 2009, ARA\&A, 47, 481

Aurière, M., Wade, G. A., Silvester, J., et al. 2007, A\&A, 475, 1053

Baglin, A. 2006, The CoRoT mission, pre-launch status, stellar seismology and planet finding, eds. M. Fridlund, A. Baglin, J. Lochard, \& L. Conroy, ESA SP-1306, 537

Bagnulo, S., Landolfi, M., Landstreet, J. D., et al. 2006, PASP, 121, 993

Balona, L. A. 1975, MNRAS, 78, 51

Balona, L. A. 1994, MNRAS, 268, 119

Battinelli, P., Brandimarti, A., \& Capuzzo-Dolcetta, R. 1994, A\&AS, 104, 379 Bessel, M. S., Castelli, F., \& Plez, B. 1998, A\&A, 333, 231 
Borra, E. F., Edwards, G., \& Mayor, M. 1984, ApJ, 284, 211

Brott, I., de Mink, S. E., Cantiello, M., et al. 2011, A\&A, 530, A115 Breger, M. 1993, A\&A, 271, 482

Bychkov, V. D., Bychkova, L. V., \& Madej, J. 2009, MNRAS, 394, 1338

Cantiello, M., Langer, N., Brott, I., et al. 2009, A\&A, 499, 279

Castelli, F., Gratton, R. G., \& Kurucz, R. L. 1997, A\&A, 318, 841

Castro, N., Urbaneja, M. A., Herrero, A., et al. 2012, A\&A, 542, A79

Crowther, P. A., Lennon, D. J., \& Walborn, N. R. 2006, A\&A, 446, 279

Cunha, K., Hubeny, I., \& Lanz, T. 2006, ApJ, 647, L143

Cutri, R. M., et al. 2012, WISE All-Sky Data Release, VizieR On-line Data Catalog: II/311

Dahm, S. E., Simon, T., Proszkow, E. M., \& Patten, B. M. 2007, AJ, 134, 999

Dambis A. K. 1999, Astron. Lett., 25, 7

de la Chevrotière, A., St.-Louis, N., Moffat, A. F. J., \& the MiMeS Collaboration 2013, ApJ, 764, 171

de Mink, S. E., Langer, N., Izzard, R. G., Sana, H., \& de Koter, A. 2013, ApJ, 764, 166

Donati, J.-F., \& Landstreet, J. D. 2009, ARA\&A, 47, 333

Donati, J.-F., Semel, M., \& Rees, D. E. 1992, A\&A, 265, 669

Donati, J.-F., Semel, M., Carter, B. D., Rees, D. E., \& Collier Cameron, A. 1997, MNRAS, 291, 658

Donati, J.-F., Howarth, I. D., Jardine, M. M., et al. 2006, MNRAS, 370, 629

Ferrario, L., Pringle, J. E., Tout, C. A., \& Wickramasinghe, D. T. 2009, MNRAS, 400, L71

Fitzpatrick, E. L. 1999, PASP, 111, 63

Flaccomio, E., Micela, G., Sciortino, S., et al. 2000, A\&A, 355, 651

Flaccomio, E., Micela, G., \& Sciortino, S. 2006, A\&A, 455, 903

Fossati, L., Ryabchikova, T., Bagnulo, S., et al. 2009, A\&A, 503, 945

Glagolevskij, Y. V. 1994, Bulletin of the Special Astrophysics Observatory, 38, 152

Glebbeek, E., Gaburov, E., Portegies Zwart, S., \& Pols, O. R. 2013, MNRAS, 434,3497

\section{Gray D.F. 1965, AJ, 70, 362}

Grevesse, N., Noels, A., \& Sauval, A. J. 1996, ASP Conf. Ser., 99, 117

Grunhut, J. H., Wade, G. A., Leutenegger, M., et al. 2013, MNRAS, 428, 1686

Hareter, M., Reegen, P., Kuschnig, R., et al. 2008, CoAst, 156, 48

Hauck, B., \& Mermilliod, M. 1997, A\&AS, 129, 431

Hempel, M., \& Holweger, H. 2003, A\&A, 408, 1065

Hibbert, A. 1988, Phys. Scr., 38, 37

Hohle, M. M., Neuhäuser, R., \& Schutz, B. F. 2010, AN, 331, 349

Kaiser, A. 2006, in Determination of Fundamental Parameters with Stroemgren Photometry, eds. C. Aerts, \& C. Sterken, ASP Conf. Ser., 349, 257

Kallinger, T., Reegen, P., \& Weiss, W. W. 2008, A\&A, 481, 571

Kharchenko, N. V., Piskunov, A. E., Röser, S., Schilbach, E., \& Scholz, R.-D. 2004, AN, 325, 740

Kharchenko, N. V., Piskunov, A. E., Röser, S., Schilback, E., \& Scholz, R.-D. 2005, A\&A, 438, 1163

Kochukhov, O. 2007, in Magnetic Stars 2006, eds. I. I. Romanyuk, D. O. Kudryavtsev, O. M. Neizvestnaya, \& V. M. Shapoval, Proc. Int. Conf., 109,118

Kochukhov, O., Makaganiuk, V., \& Piskunov, N. 2010, A\&A, 524, A5

Kochukhov, O., Lundin, A., Romanyuk, I., \& Kudryavtsev, D. 2011, ApJ, 726, 24

Korntreff, C., Kaczmarek, T., \& Pfalzner, S. 2012, A\&A, 543, A126

Kunzli, M., North, P., Kurucz, R. L., \& Nicolet, B. 1997, A\&AS, 122, 51

Kupka, F., Piskunov, N., Ryabchikova, T. A., Stempels, H. C., \& Weiss, W. W. 1999, A\&AS, 138, 119

Kuschnig, R., Weiss, W. W., Gruber, R., Bely, P. Y., \& Jenkner, H. 1997, A\&A, 328,544

Langer, N. 2012, A\&ARA, 50, 107

Langer, N. 2013, in Proc. IAU Symp., 302, Magnetic fields throughout stellar evolution, in press [arXiv: 1312.2373]

Lata, S., Pandey, A. K., Sagar, R., \& Mohan, V. 2002, A\&A, 388, 158

Lefever, K. 2007, Ph.D. Thesis, K.U. Leuven

Lenz, P., \& Breger, M. 2005, CoAst, 146, 53

Liu, T., Janes, K. A., \& Bania, T. M. 1991, AJ, 102, 1103

Loktin, A. V., Gerasimenko, T. P., \& Malysheva, L. K. 2001, Astron. Astrophys. Trans., 20, 607

Lunga, G. 1985, Computer-Based Catalogue of Open Cluster Data, eds. van Woerden, H., Allen, R. J., \& Burton, W. B., IAU Symp., 106, 143
Maeder, A., \& Meynet, G. 2012, Rev. Mod. Phys., 84, 25

Malysheva, L. K. 1997, Astron. Lett., 23, 585

Martins, F., Escolano, C., Wade, G. A., et al. 2012, A\&A, 538, A29

Massey, P., Neugent, K. F., Hillier, D. J., \& Puls, J. 2013, ApJ, 768, 6

Mermilliod, J. C. 1991, Catalogue of Homogeneous Means in the UBV System, VizieR On-line Data Catalog: II/168

Mermilliod, J.-C., \& Paunzen, E. 2003, A\&A, 410, 511

Michaud, G. 1970, ApJ, 160, 641

Michaud, G., Martel, A., Montmerle, T., et al. 1979, ApJ, 234, 206

Moon, T. T., \& Dworetsky, M. M. 1985, MNRAS, 217, 305

Morel, T., \& Butler, K. 2008, A\&A, 487, 307

Morel, T., Briquet, M., Auvergne, M, et al. 2014, A\&A, 561, A35

Morgan, W. W., Hiltner, W. A., Neff, J. S., Garrison, R., \& Osterbrock, D. E. 1965, ApJ, 142, 974

Moss, D. 2001, Magnetic Fields in the Ap and Bp Stars: a Theoretical Overview, Magnetic Fields Across the Hertzsprung-Russell Diagram, eds. G. Mathys, S. K. Solanki, D. T. Wickramasinghe, ASP Conf. Ser., 248, 305

Napiwotzki, R., Schoenberner, D., \& Wenske, V. 1993, A\&A, 268, 653

Piskunov, N. E., Kupka, F., Ryabchikova, T. A., Weiss, W. W., \& Jeffery, C. S. 1995, A\&AS, 112, 525

Przybilla, N., Nieva, M.-F., \& Butler, K. 2008, ApJ, 688, L103

Puls, J., Urbaneja, M. A., Venero, R., et al. 2005, A\&A, 435, 669

Puls, J., Vink, J. S., \& Najarro, F. 2008, A\&ARv, 16, 209

Raskin, G., van Winckel, H., Hensberge, H., et al. 2011, A\&A, 526, A69

Reegen, P. 2007, A\&A, 467, 135

Renson, P., \& Manfroid, J. 2009, A\&A, 498, 961

Rufener, F., Hauck, B., Goy, G., Peytremann, E., \& Maeder, A. 1996, Journal des Observateurs, 49, 417

Ryabchikova, T. A., Piskunov, N. E., Stempels, H. C., Kupka, F., \& Weiss, W. W. 1999, Phis. Scr., T83, 162

Salpeter, E. E. 1955, ApJ, 121, 161

Sana, H., de Mink, S. E., de Koter, A., et al. 2012, Science, 337, 444

Santolaya-Rey, A. E., Puls, J., \& Herrero, A. 1997, A\&A, 323, 488

Schaerer, D., \& Schmutz, W. 1994, A\&A, 288, 231

Schneider, F. R. N., Langer, N., Lau, H. H. B., \& Izzard, R. G. 2013, The BONNSAI project: A statistical comparison of individual stars with stellar evolution models, Setting a new standard in the analysis of binary stars, Leuven, Belgium, in press

Schneider, F. R. N., Izzard, R. G., de Mink, S. E., et al. 2014, ApJ, 780, 117

Shulyak, D., Tsymbal, V., Ryabchikova, T., Stütz Ch., \& Weiss, W. W. 2004, A\&A, 428, 993

Simón-Díaz, S., \& Herrero, A. 2007, A\&A, 468, 1063

Simón-Díaz, S., \& Herrero, A. 2014, A\&A, 562, A135

Spassova N. M., \& Beav P. V. 1985, Astrophys. Space Sci., 112, 111

Sung, H., Bessel, M. S., \& Lee, S.-W. 1997, AJ, 114, 2644

Sung, H., Sauffer, J. R., \& Bessel, M. S. 2009, AJ, 138, 1116

Thompson, G. I., Nandy, K., Jamar, C., et al. 1978, Catalogue of stellar ultraviolet fluxes (TD1): A compilation of absolute stellar fluxes measured by the Sky Survey Telescope (S2/68) aboard the ESRO satellite TD-1, VizieR Online Data Catalog: II/59B

van der Bliek, N. S., Manfroid, J., \& Bouchet, P. 1996, A\&AS, 119, 547

Vick, M., Michaud, G., Richer, J., \& Richard, O. 2010, A\&A, 521, A62

Vogel, S. N., \& Kuhi, L. V. 1981, ApJ, 245, 960

Wade, G. A., Grunhut, J. H., \& MiMeS Collaboration 2012, The MiMeS Survey of Magnetism in Massive Stars in Circumstellar Dynamics at High Resolution, eds A. C. Carciofi, \& T. Rivinius, ASP Conf. Ser., 464, 405

Wade, G. A., Grunhut, J., Alecian, E., et al. 2013, The magnetic characteristics of Galactic OB stars from the MiMeS survey of magnetism in massive stars, Proceedings of IAUS 302: Magnetic fields throughout stellar evolution, in press [arXiv: 1310.3965]

Walker, M. F. 1956, ApJS, 2, 365

Walker, G., Matthews, J. M., Kuschnig, R., et al. 2003, PASP, 115, 1023

Weisskopf, M. C., Brinkman, B., Canizares, S., et al. 2002, PASP, 114, 1

Werner, M. W., Roellig, T. L., Low, F. J., et al. 2004, ApJS, 154, 1

Wright, E. L., Eisenhardt, P. R. M., Mainzer, A. K., et al. 2010, AJ, 140, 1868

Zacharias, N., Monet, D. G., Levine, S. E., et al. 2005, BAAS, 36, 1418

Zwintz, K., Hareter, M., Kuschnig, R., et al. 2009, A\&A, 502, 239

Zwintz, K., Fossati, L., Ryabchikova, T., et al. 2013a, A\&A, 550, A121

Zwintz, K., Fossati, L., Guenther, D. B., et al. 2013b, A\&A, 552, A68 